\title{
Performance of Large Spacer Versus Nebulizer T-Piece in Single-Limb Noninvasive Ventilation
}

\author{
Hadeer S Harb, Ahmed A Elberry PhD, Hoda Rabea PhD, Maha Fathy PhD, and \\ Mohamed EA Abdelrahim PhD
}

\begin{abstract}
BACKGROUND: Predosing patients with COPD with salbutamol by using a pressurized metereddose-inhaler (pMDI) as a bronchodilator was hypothesized to improve the distribution of the subsequent nebulized dose. This study determined the effect of a pMDI preliminary bronchodilator dose on the aerosol delivered by a mesh nebulizer during single-limb noninvasive ventilation. METHODS: Twelve subjects with COPD who received noninvasive ventilation were enrolled in a randomized, open-label, urinary pharmacokinetic study. A bi-level ventilator with a dry single-limb circuit and the fixed expiratory port was set in the spontaneous mode, with initial inspiratory and expiratory pressures of 20 and $5 \mathrm{~cm} \mathrm{H}_{2} \mathrm{O}$ respectively, a 1:3 inspiratory-expiratory ratio, and 15 breaths $/ \mathrm{min}$. Salbutamol was administered via a mesh nebulizer with a large spacer or T-piece placed between the fixed-orifice expiratory valve and the oronasal mask. In vivo dosing methods were randomized for days $\mathbf{1 , 3}$, and 5 of the study. On each day, a 1-mL respirable solution that contained 5,000 $\mu \mathrm{g}$ salbutamol was nebulized by using a mesh nebulizer with 3 setting: (1) T-piece, (2) large spacer, and (3) large spacer plus pMDI. Only with the large spacer plus pMDI setting, 2 pMDI doses, which contained $100 \mu \mathrm{g}$ salbutamol each, were actuated before nebulization. Urine samples were collected at $0.5 \mathrm{~h}$ (as an index of pulmonary bioavailability) and pooled up to $24 \mathrm{~h}$ after dosing (as an index of systemic absorption). On day 2 , ex vivo studies were performed for the 3 setting with salbutamol collected onto filters placed before the mask. The drug was eluted from the filters and analyzed to determine the inhaled dose. RESULTS: A large spacer plus pMDI showed a trend to deliver a higher fraction (percentage of nominal dose) of both ex vivo filters and 0.5-h urinary salbutamol. The 0.5-h urinary salbutamol excreted with a large spacer plus pMDI (1.99\%) was larger than with the T-piece $(1.73 \%)$ and large spacer $(1.78 \%)$. This trend did not extend to the 24-h levels, in which bioavailability with the large spacer plus pMDI (49.9\%) was lower than with the T-piece (52.8\%) and with the large spacer $(\mathbf{5 4 . 3 \%})$. However, no differences were significant. CONCLUSIONS: The T-piece and large spacer were equally efficient for salbutamol delivery from the mesh nebulizer in patients with COPD and on single-limb noninvasive ventilation. Adding a preliminary bronchodilator dose by pMDI prenebulization showed a trend toward greater pulmonary bioavailability of nebulized salbutamol and may be worth considering to maximize delivery of salbutamol to patients who are severely ill. Key words: pMDI; salbutamol; NIV; COPD; lung; bioavailability nebulizer. [Respir Care 2018;63(11):1360-1369. ( 2018 Daedalus Enterprises]
\end{abstract}

Introduction

Medical aerosols are commonly administered to patients with COPD during exacerbations that require ven-

\footnotetext{
Ms Harb, Dr Rabea, and Dr Abdelrahim are affiliated with the Clinical Pharmacy Department, Faculty of Pharmacy, Beni-Suef University, BeniSuef, Egypt. Dr Elberry is affiliated with the Clinical Pharmacology Department, Faculty of Medicine, Beni-Suef University, Beni-Suef, Egypt. Dr Fathy is affiliated with the Respiratory Department, Faculty of Medicine, Beni-Suef University, Beni-Suef, Egypt. Dr Abdelrahim is affili-
}

tilator support. Patients with COPD often have irregular aerosol distribution in the lungs due to inflammation

\footnotetext{
ated with the Clinical Pharmacy Department, Faculty of Pharmacy, Modern University for Technology and information, Cairo, Egypt.

Controllers and vibrating mesh nebulizers were supplied by Aerogen. Combihaler spacers were supplied by Laboratoire ProtecSom.

Dr Abdelrahim discloses a relationship with Aerogen. The other authors have disclosed no conflicts of interest.
} 
and bronchoconstriction, ${ }^{1-3}$ although aerosol deposition is higher in patients with COPD than those without. ${ }^{4}$ We hypothesized that initial administration of 2 puffs of a fast-acting $\beta_{2}$ agonist bronchodilator from a pressurized metered-dose-inhaler (pMDI) may provide an initial airway response that would improve the distribution of subsequently administered nebulized drug. This would be evidenced by a greater proportion of the dose absorbed from the lung and assayed in the urine. In addition, adapters commonly used to administer medical aerosols to patients who require mechanical ventilation impact delivery efficiency based by their design and placement.

Placing relatively large-volume spacers in the inspiratory limb of a conventional ventilator circuit proximal to the patient has improved the inhaled dose with continuous aerosol generators, such as jet, ultrasonic, and vibrating mesh nebulizers. The large-volume reservoir acts to collect aerosol between inspirations, which makes as much as $35 \%$ more aerosol available distal to the endotracheal tube. ${ }^{5}$ Similarly, Boukhettala et $\mathrm{al}^{6} \mathrm{dem}-$ onstrated that add-on devices could improve aerosol delivery from vibrating mesh nebulizers and hypothesized that larger spacers could conserve more aerosol during the expiration phase. ${ }^{7}$

CombiHaler (Laboratoire ProtecSom, Valognes, France) is a large-volume $(210 \mathrm{~mL})$ spacer chamber designed to administer aerosols through the ventilator circuit with both pMDIs and vibrating mesh nebulizers (Aerogen Solo, Aerogen, Galway, Ireland). Aerosol emitted by the vibrating mesh nebulizer into the large spacer has a distance of $70 \mathrm{~mm}$ between the aerosol-generating mesh and the interior wall of the spacer. ${ }^{6}$ In contrast, the standard T-piece has an internal volume of $35 \mathrm{~mL}$ and distance from the mesh to the opposing internal wall of $35 \mathrm{~mm}$. The internal volume of the spacer acts as a reservoir that collects aerosol between breaths, that makes more available with the next breath; the greater the distance from the aerosol-generating mesh to an opposing wall, the lower the proportion of aerosol particles that impact en route to the ventilator circuit.

During invasive mechanical ventilation with a 2-limb circuit, the large spacer has been shown to deliver a larger inhaled dose than the T-piece for aerosol delivery from vibrating mesh nebulizers. ${ }^{6,8,9}$ However, those findings may not be relevant to use in a single-limb circuit

Correspondence: Mohamed EA Abdelrahim PhD, Department of Clinical Pharmacy, Modern University for Technology and information, Al Abageyah, Qism El-Khalifa, 12055 Cairo, Egypt. E-mail: mohamedemam9@yahoo.com.

DOI: $10.4187 /$ respcare.05976

\section{QUICK LOOK}

\section{Current knowledge}

The CombiHaler (large spacer) is a large-volume spacer designed to connect both pressurized metered-dose inhalers and vibrating mesh nebulizers into conventional and noninvasive ventilation circuits. Adapters used to administer aerosol therapy during mechanical ventilation impact delivery based on design and placement in the circuit. Optimum device placement is different with invasive and non-invasve ventilation.

\section{What this paper contributes to our knowledge}

A vibrating mesh nebulizer standard T-piece and large spacer were equally efficient for salbutamol delivery from a vibrating mesh nebulizer in subjects with COPD on single-limb NIV. Adding a preliminary bronchodilator dose prenebulization could improve the inhaled salbutamol fraction delivered to the lungs in the first 30 min by enhancing peripheral penetration of the aerosol. However, the effect was not observed at $24 \mathrm{~h}$, and differences were not statistically significant.

with a noninvasive ventilator, in which higher continuous gas flow passes through a fixed orifice resistor. In addition, the patient interface (mask vs endotracheal tube) and placement of the aerosol generator differ with noninvasive ventilation (NIV) compared with conventional invasive ventilation. ${ }^{10-13}$

In a previous in vitro study, we reported that the standard T-piece and large spacer were equally efficient for salbutamol delivery by a vibrating mesh nebulizer in a single-limb NIV under nonhumidified conditions. ${ }^{14}$ In vitro methods that study the aerodynamic characteristics and fate of aerosol by using a lung simulator model are thought to offer some degree of guidance to clinical delivery and response. However, inhaled doses determined in vitro do not accurately account for the variability of breath parameters in vivo, and the lung dose in vivo is lower than filters because lungs exhale more aerosol than do collecting filters. ${ }^{15}$ In vitro studies often use respiratory parameters that may not reflect variations encountered in patients. Ex vitro methods collect aerosol on filters placed between the ventilator circuit and the subject during NIV, which better represents actual breathing parameters of each subject.

We hypothesize that predosing with pMDI would increase inhaled drug delivered ex vivo and the proportion of drug recovered in vivo. This supports the case to establish ex vitro-in vivo correlation. ${ }^{16-23}$ This in vivo study with an ex vivo component compared aerosol delivery by a vibrating mesh nebulizer by using standard T-piece and 


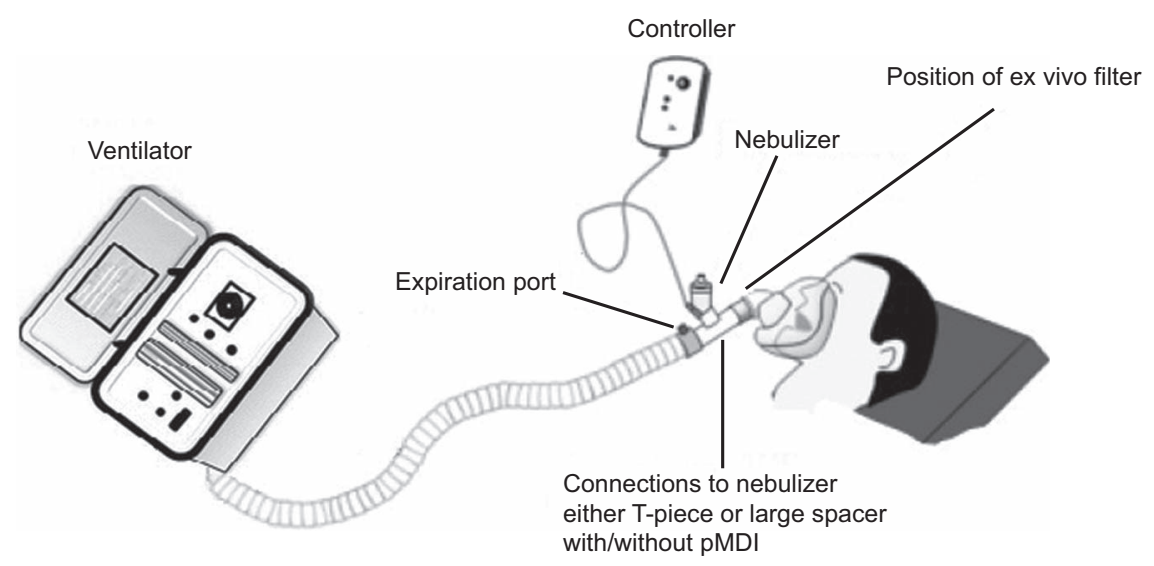

Fig. 1. Schematic design of in vivo and ex vivo single-limb noninvasive ventilation setup.

by using large-spacer adapter, and determined the possible effect of preliminary bronchodilator dose with a pMDI on the delivered nebulizer dose when using a single-limb circuit NIV in subjects with COPD.

\section{Methods}

\section{Study Design}

The in vivo study was an experimental, randomized, open-label study with a crossover design.

\section{Inclusion and Exclusion of Subjects}

The study included 12 subjects ( 6 women) with a previous diagnosis of COPD who had been admitted in the chest department at Beni-Suef University Hospital and Hospital of Chest with an exacerbation that required NIV as prescribed by their attending physician. Local hospital research ethics committee approval (FM-BSU REC FWA: FWA00015574) was obtained. Subjects with an exacerbation of COPD and with normal renal function who required NIV support for respiratory acidosis prescribed to receive nebulized salbutamol were included. Inclusion approval was taken under the care of a consulting chest physician. Patients were ineligible to participate if they had taken part in a research study during the previous 6 months, had a known hypersensitivity to salbutamol, systolic blood pressure of $<100 \mathrm{~mm} \mathrm{Hg}$, or severe renal impairment defined as creatinine clearance or estimated glomerular filtration rate of $<20 \mathrm{~mL} / \mathrm{min}$.

\section{Study Protocol}

This study was conducted in accordance with the amended Declaration of Helsinki. Written informed consent was obtained from all the subjects. A washout period of 12-24 h allowed the body to excrete salbutamol delivered before the study. During the course of the study, prescribed salbutamol treatments were substituted with $25 \mu \mathrm{g} / \mathrm{mL}$ ipratropium bromide aerosol (Boehringer Ingelheim, Ingelheim am Rhein, Germany). The subjects participated in the study for 6 days, in which they received 3 salbutamol study doses. The choice of inhaled salbutamol dosage was in accordance with previous literature. ${ }^{16,18,24-36}$ As recommended previously in studies that used single-limb NIV, ${ }^{10,30}$ the nebulizers were placed proximal to the subject and distal to the fixed expiratory valve, as shown in Figure 1.

The subjects were randomized to receive the salbutamol dosing iterations in a crossover design on days 1 , 3 , and 5. A washout period of $48 \mathrm{~h}$ was provided between each salbutamol dosing. ${ }^{37}$ The subjects received nebulized doses of $1 \mathrm{~mL}$ of salbutamol respirable solution that contained a nominal dose of $5,000 \mu \mathrm{g} / \mathrm{mL}$ salbutamol (Pharco Pharmaceuticals, Alexandria, Egypt) by using an Aerogen Solo vibrating mesh nebulizer (Aerogen) powered by the Aerogen Pro-X Controller with a standard T-piece and CombiHaler (large spacer) positioned in the single-limb NIV circuit between the fixed valve and the subject (Fig. 2). A 1-mL respirable solution that contained 5,000 $\mu \mathrm{g}$ salbutamol was nebulized by using a mesh nebulizer with 3 connection methods: (1) T-piece, (2) large spacer, (3) large spacer plus pMDI. Only with the large spacer plus pMDI group, 2 pMDI doses, which contained $100 \mu \mathrm{g}$ salbutamol each (Ventoline, GlaxoSmithKline, Brentford, United Kingdom) synchronized with the beginning of subject inspiration, with $30 \mathrm{~s}$ between actuations, as a preliminary bronchodilator dose, followed by nebulization via a vibrating mesh nebulizer.

A bi-level ventilator (B\&D Electromedical, StratfordUpon-Avon, United Kingdom) with a dry single-limb 


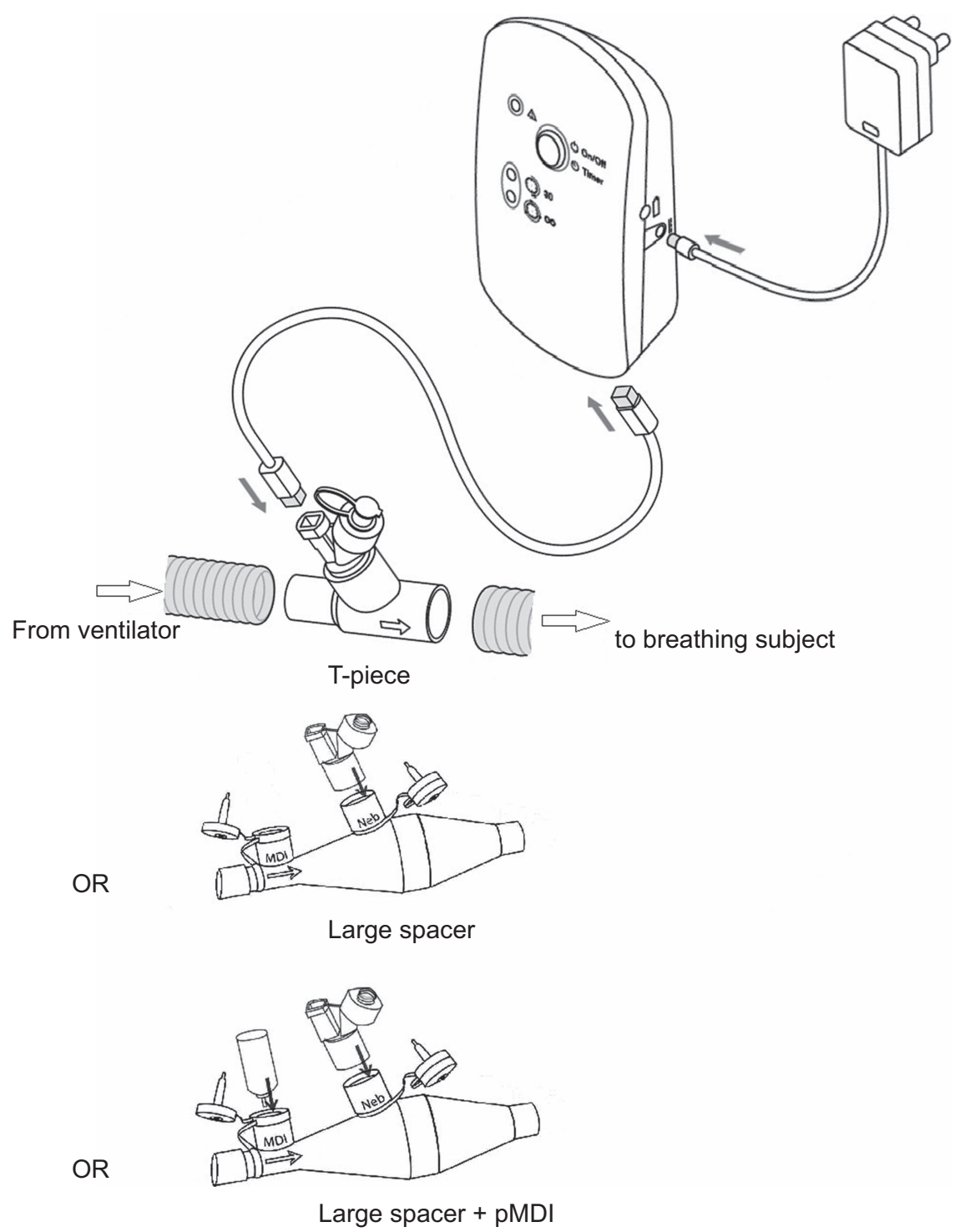

Fig. 2. Assembly of aerosol delivery system and its connections; all 3 connections incorporated the vibrating mesh nebulizer.

circuit and fixed orifice expiratory port was set in the spontaneous mode with initial inspiratory and expiratory pressures of 20 and $5 \mathrm{~cm} \mathrm{H}_{2} \mathrm{O}$, respectively $1: 3$ inspiratoryexpiratory ratio, 15 breaths/min, when using a wellfitted patient mask (Respironics AF531 oronasal mask, Philips Respironics, Murrysville, Pennsylvania). The subjects received pressure support ventilation with a PEEP of $5 \mathrm{~cm} \mathrm{H}_{2} \mathrm{O}$. The subjects' spontaneous tidal volumes were monitored, and a clinician titrated the inspiratory pressure support to reach the targeted tidal volume of $500 \mathrm{~mL}$. The inspiratory pressures in pressure support ventilation ranged between 15 and $20 \mathrm{~cm} \mathrm{H}_{2} \mathrm{O}$. A vibrating mesh nebulizer with a T-piece and large spacer was placed in the ventilator circuit proximal to subjects' oronasal mask.
The subjects voided their urine 15 min before each study dose and then were asked to provide a urine sample $30 \mathrm{~min}$ after the start of the nebulization (USAL0.5) as an index of salbutamol delivery to the lungs. ${ }^{37}$ The subjects collected all their urine into a container over the next $24 \mathrm{~h}$ (USAL24) as an index of systemic absorption of salbutamol after inhalation. ${ }^{37}$ The urine sample volumes were measured, and aliquots were retained for salbutamol content solid-phase extraction with an Oasis MCX $60 \mathrm{mg}, 10 \mathrm{~mL}$ XL cartridge (Waters Corporation, Milford, Massachusetts) with the application of a low vacuum ( $<3$ bars) through a VAC-ELUT 10 manifold (Varian, Palo Alto, California) extraction station. ${ }^{36}$ The salbutamol amount in each extract was determined by using high-performance liquid chromatography, described in the High-Performance Liqud Chromatography Analysis. 


\section{Ex Vivo Protocol}

On day 2 of the study, a collecting filter (Filta Guard breathing filter, Intersurgical, Wokingham, United Kingdom) was placed between the nebulizer and the adapter, and the subject's mask (AF531 oronasal mask, Philips Respironics) so that the subjects would not inhale the drug, as shown in Figure 1. The same study dose and/or adapters were administered to each subject, with filters changed with each administration. Salbutamol entrained on the filter was desorbed by sonication with $25 \mathrm{~mL}$, $30 \%$ acetonitrile before rinsing, to be analyzed by using the high-performance liquid chromatography method with ultraviolet detection, described in High-Performance Liqud Chromatography Analysis.

\section{Solid-Phase Extraction}

Urine samples 30 min postdose were prepared by acidification with $2 \mathrm{~mL} 0.5 \mathrm{~N} \mathrm{HCL}$ for each $10 \mathrm{~mL}$ of urine, whereas urine samples $24 \mathrm{~h}$ postdose used acid hydrolysis of salbutamol ester conjugate by adding $2 \mathrm{~mL}$ $0.1 \mathrm{~N} \mathrm{HCL}$ for each $10 \mathrm{~mL}$ urine. The sample was mixed well by shaking, then vortexed, covered with aluminum foil, boiled in a water bath at $100^{\circ} \mathrm{C}$ for $5 \mathrm{~min}$, and then cooled and filtered. ${ }^{38,39}$

Solid-phase extraction protocol steps included conditioning the cartridge with $6 \mathrm{~mL}$ methanol, then equilibrated with $6 \mathrm{~mL}$ water before loading with $10 \mathrm{~mL}$ of the prepared urine sample, followed by several washes to remove any possible urine from the extract. The washes included $10 \mathrm{~mL} \mathrm{5 \%} \mathrm{methanol} \mathrm{in} 0.1 \mathrm{~N} \mathrm{HCL}, 10 \mathrm{~mL}$ methanol and $6 \mathrm{~mL} \mathrm{2.5 \%}$ triethanolamine in methanol and finally elution by $10 \mathrm{~mL} \mathrm{5 \%}$ ammonium hydroxide in methanol. ${ }^{36}$

\section{High-Performance Liqud Chromatography Analysis}

Urine Samples. The high-performance liquid chromatography system was composed of an ODS $5 \mu \mathrm{m}$ $(4.6 \times 250 \mathrm{~mm}$, ZORBAX Eclipse, Agilent, Santa Clara, California) C18 high-performance liquid chromatography column with a $4 \times 3 \mathrm{~mm}$ (Agilent) $\mathrm{C} 18$ (ODS) guard column. The mobile phase, which contains acetonitrile-water $(90: 10 \mathrm{v} / \mathrm{v})$, was pumped through the columns at a flow of $1 \mathrm{~mL} / \mathrm{min}$ maintained at $25^{\circ} \mathrm{C}$ and photodiode array detection was set at $220 \mathrm{~nm}$. The lower limit of detection and lower limit of quantification for salbutamol were 0.36 and $1.00 \mu \mathrm{g} / \mathrm{mL}$, respectively. Calibration solutions ranged from 8 to $70 \mu \mathrm{g} / \mathrm{mL}$ (wt/v) was used in the construction of a calibration curve to interpret sample concentrations.

Aqueous Samples of Ex Vivo Filters. The system was composed of an ODS $5 \mu \mathrm{m}(4.6 \times 250 \mathrm{~mm}$, ZORBAX Eclipse) C-18 high-performance liquid chromatography column. The mobile phase, which contained (90:10, v/v) acetonitrile-water, was pumped through the columns at a flow of $1 \mathrm{~mL} / \mathrm{min}$ maintained at $25^{\circ} \mathrm{C}$ and photodiode array detection was set at $225 \mathrm{~nm}$. The lower limit of detection was $0.35 \mu \mathrm{g} / \mathrm{mL}$, and the lower limit of quantification was $2.55 \mu \mathrm{g} / \mathrm{mL}$. Calibration solutions, which range from 10 to $100 \mu \mathrm{g} / \mathrm{mL}(\mathrm{wt} / \mathrm{v})$, were used in the construction of a calibration curve to interpret samples concentrations.

\section{Statistical Analysis of Data}

All data are expressed as mean \pm SD. Two-way analysis of variance with the application of least significant difference correction was used to compare both the urinary excretion of salbutamol and salbutamol on ex vivo filters postinhalation through the 3 different nebulizer connections by using SPSS V17.0 (SPSS, Chicago, Illinois), with $P<.05$ considered significant.

\section{Results}

Twelve subjects with COPD (6 women) were screened, had consented, and had completed the protocol, with mean $\pm \mathrm{SD}$ age, weight, and height of $63.3 \pm 8.5 \mathrm{y}$, $85.42 \pm 10.17 \mathrm{~kg}$, and $165.92 \pm 6.33 \mathrm{~cm}$, respectively. The mean \pm SD amount $(\mu \mathrm{g})$ and proportion of nominal dose (\%) found in USAL0.5 and USAL24, and ex vivo salbutamol from filters for the T-piece, the large spacer, and the large spacer plus pMDI are shown in Table 1 and Figures 3-5, respectively.

\section{USAL0.5}

The amount of salbutamol with the large spacer plus pMDI showed a trend toward $16 \%$ and $18 \%$ higher than the T-piece and the large spacer, respectively, but no differences were significant with the $P$ values of comparison between the T-piece versus the large spacer; the T-piece versus the large spacer plus the pMDI, and the large spacer versus the large spacer plus the pMDI were $P=.87$, $P=.35$, and $P=.43$, respectively.

\section{USAL24}

Large spacer and large spacer plus pMDI were nearly equal, as shown in Table 1 and Figure 4. The $P$ values between the T-piece versus the large spacer; the T-piece versus the large spacer plus pMDI and large spacer versus large spacer plus pMDI was $P=.85, P=.89$, and $P=.96$, respectively (Fig. 4). 
Table 1. The Nominal Dose of Salbutamol on Ex Vivo Filters and at USAL0.5 and USAL24 After Inhalation of Salbutamol Respirable Solution Via Vibrating Mesh Nebulizer Through the 3 Different Connections

\begin{tabular}{|c|c|c|c|}
\hline Dose & T-Piece & Large Spacer & Large Spacer + pMDI \\
\hline \multicolumn{4}{|l|}{ USAL0.5, mean $\pm \mathrm{SD}$} \\
\hline$\mu \mathrm{g}$ & $86.56 \pm 33.04$ & $88.86 \pm 25.32$ & $103.43 \pm 38.60$ \\
\hline$\%$ & $1.73 \pm 0.66$ & $1.78 \pm 0.51$ & $1.99 \pm 0.74$ \\
\hline \multicolumn{4}{|l|}{ USAL24, mean \pm SD } \\
\hline$\mu \mathrm{g}$ & $599.84 \pm 99.06$ & $610.27 \pm 159.88$ & $631.69 \pm 133.39$ \\
\hline$\%$ & $12.00 \pm 1.98$ & $12.21 \pm 3.20$ & $12.15 \pm 2.57$ \\
\hline \multicolumn{4}{|l|}{ SALF (ex-vivo inhaled dose), mean \pm SD } \\
\hline$\mu \mathrm{g}$ & $1106.15 \pm 302.17$ & $1155.66 \pm 311.84$ & $1265.22 \pm 296.43$ \\
\hline$\%$ & $22.12 \pm 6.04$ & $23.11 \pm 6.24$ & $24.33 \pm 5.70$ \\
\hline USAL2 4 as a percentage of SALF, $\%$ & 54.3 & 52.8 & 49.9 \\
\hline $\begin{array}{l}N=12 . \\
\text { pMDI = pressurized metered-dose inhaler } \\
\text { USAL0.5 = urinary salbutamol at } 0.5 \mathrm{~h} \text { after administration } \\
\text { USAL24 = urinary salbutamol over } 24 \mathrm{~h} \text { after administration } \\
\text { SALF = Salbutamol collected on filter }\end{array}$ & & & \\
\hline
\end{tabular}

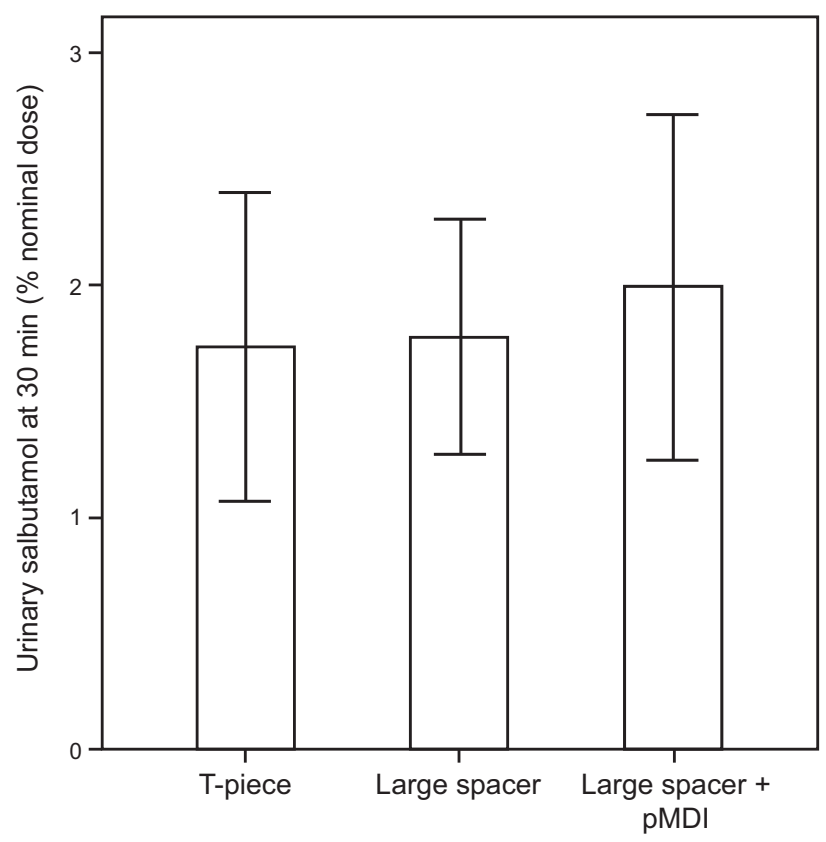

Fig. 3. The mean \pm SD urinary excretion of salbutamol $30 \mathrm{~min}$ after inhalation via vibrating mesh nebulizer through the 3 different connections expressed as the percentage of the nominal dose $(N=12)$. The $P$ values of the comparison between the T-piece vs the large spacer; the T-piece vs the large spacer plus the pressurized metered-dose-inhaler (pMDI), and the large spacer vs the large spacer plus pMDI were $P=.87, P=.35$, and $P=.43$, respectively.

\section{Ex Vivo}

The inhaled dose was similar with the T-piece and the large spacer with an incremental increase associated with the 2 puffs by using a pMDI, but the differences were not

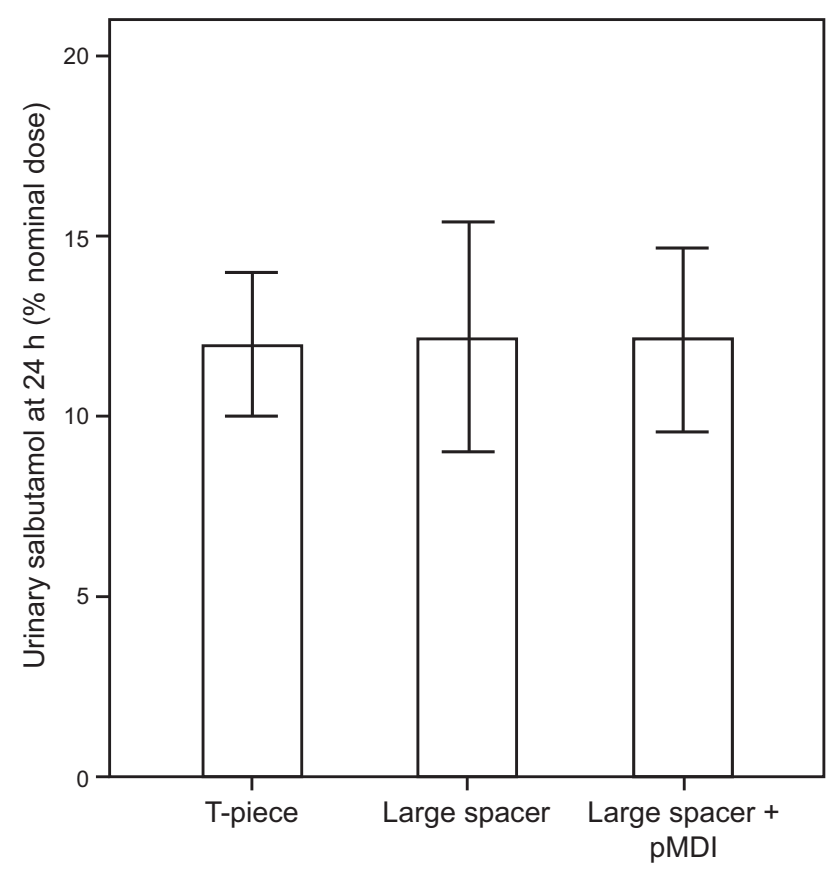

Fig. 4. The mean \pm SD urinary excretion of salbutamol $24 \mathrm{~h}$ after inhalation via the vibrating mesh nebulizer through the 3 different connections expressed as percentages of the nominal dose $(N=12)$. The $P$ values of comparison between the T-piece vs the large spacer, the T-piece vs the large spacer plus pressurized metered-dose-inhaler (pMDI), and the large spacer vs the large spacer plus pMDI were $P=.85, P=.90$, and $P=.96$, respectively.

significant. The $P$ values of comparison between the Tpiece versus the large spacer, the T-piece versus the large spacer plus the $\mathrm{pMDI}$, and the large spacer versus the large spacer plus the pMDI were $P=.70, P=.39$, and $P=.63$, respectively. 


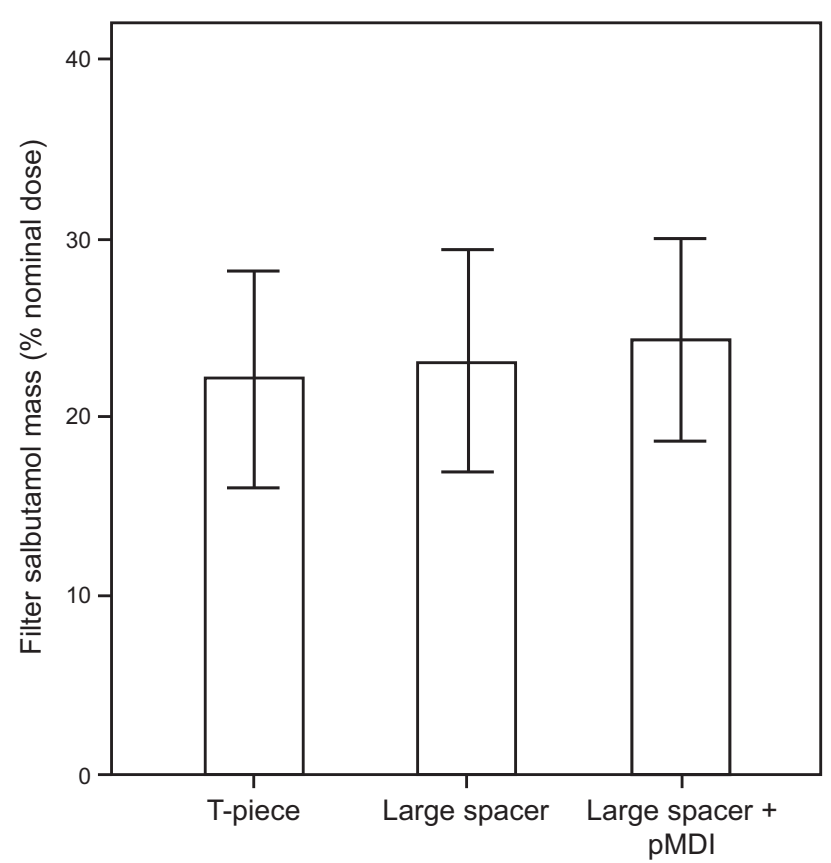

Fig. 5. The mean \pm SD amount of salbutamol recovered from the ex vivo filter after nebulization via the vibrating mesh nebulizer through the 3 different connections expressed as percentages of the nominal dose $(N=12)$. The $P$ values of comparison between the T-piece vs the large spacer, the T-piece vs the large spacer plus pressurized metered-dose-inhaler (pMDI) and the large spacer vs the large spacer plus the pMDI were $P=.70, P=.39$, and $P=.63$, respectively.

\section{Discussion}

This study was the first to compare ex vivo inhaled dose to in vivo urinary drug levels after medical aerosol delivery from a nebulizer and a pMDI during NIV with a single-limb circuit. These findings were consistent with previous in vitro work to assess the performance of a vibrating mesh nebulizer in single-limb NIV. ${ }^{14}$ Our ex vivo findings were consistent with in vitro testing with both vibrating mesh nebulizer with the T-piece and the pMDI during NIV in a study by AlQuaimi et al. ${ }^{40}$

Similarly Galindo-Filho et $\mathrm{al}^{4}$ administered radiolabeled aerosol via a vibrating mesh nebulizer to 10 healthy subjects during NIV with inspiratory and expiratory pressures $12 / 5 \mathrm{~cm} \mathrm{H}_{2} \mathrm{O}$ by using an oronasal face mask. They reported an inhaled dose of $23.1 \%$ and a lung dose of $6.5 \%$ in healthy normal subjects. They subsequently reported similar study findings in subjects with stable COPD with an inhaled dose of $19.9 \%$ and lung dose of $8.8 \% .^{4}$ This confirmed the range of the inhaled dose in vivo and hypothesis that lung dose is higher in subjects with COPD versus normal subjects and the presence of greater airway obstruction with COPD. These regions of obstruction in the airways would be a likely target for benefit from prenebulization bronchodilation with pMDI.
The urine analysis method developed by Hindle and Chrystyn, ${ }^{37}$ reflects the relative bioavailability of salbutamol to the lungs after inhalation by assessing urinary salbutamol excretion based on its absorption lag time. The amount of urinary salbutamol 30 min after inhalation represents an index of salbutamol delivery to the lungs. The amount of salbutamol excreted in urine within $24 \mathrm{~h}$ after inhalation represents an index of systemic absorption of salbutamol. ${ }^{37,41}$ Our findings were consistent with previously reported urine levels of salbutamol in a study of aerosol delivery in subjects on mechanical ventilation with salbutamol 5,000 $\mu \mathrm{g}$ via the vibrating mesh nebulizer with a T-piece in the inspiratory limb of a conventional 2-limb circuit. Moustafa et $\mathrm{al}^{36}$ reported salbutamol levels in the urine at $0.5 \mathrm{~h}$ of $1.8(1.1) \%$, with $10.5(4.0) \%$ at $24 \mathrm{~h}$. Our findings of inhaled dose percentage of $1.73 \%$ at $0.5 \mathrm{~h}$ and $12 \%$ at $24 \mathrm{~h}$ with a vibrating mesh nebulizer with a $\mathrm{T}$ piece are similar, despite the difference in the type of ventilator and circuit used. They reported that pMDI administered with a large-volume adapter had a USAL0.5\% at 2.5 (1.3) \% with USAL24\% of 8.0 (3.4) \%. The pMDI had a higher USAL $0.5 \%$ than the vibrating mesh nebulizer, but the USAL24\% was lower.

The ex vivo filter data as a percentage of the nominal dose showed a trend that the large spacer was marginally higher than the T-piece (1\%), and the addition of the 2 puffs from the pMDI with a large spacer was an additional $1.2 \%$ higher. This confirmed that a portion of the 2 puffs from the pMDI had an additive effect on the inhaled dose. We found that 2 puffs from the pMDI was reflected in the urine at $0.5 \mathrm{~h}$ after administration, with a trend to levels that seemed higher with the large spacer plus pMDI than the incremental increase shown ex vivo, which suggested that total bioavailability showed a trend toward higher with the prenebulizer administration of the pMDI. However, at $24 \mathrm{~h}$, the difference in the drug amount was lower than with the nebulizer alone, and the percentage of the nominal dose showed a trend toward lower with the pMDI. This suggested that the addition of prenebulization administration of the pMDI may have a short-term effect but did not increase total bioavailability of salbutamol at $24 \mathrm{~h}$. This was seen in the USAL24 amount because a proportion of the ex vivo inhaled dose was higher with the Tpiece $(54.3 \%)$ than with the large spacer $(52.8 \%)$ and the large spacer and the pMDI (49.9\%).

Adding a preliminary bronchodilator dose prenebulization resulted in a trend to higher USAL0.5\% than delivering the nebulizer bronchodilator dose alone. This trend could lend some support to our hypothesis. However, the short-term benefit of the pMDI did effect, not extend to the USAL24\%. This could be of benefit to decrease systemic adverse effects and at the same time increase pulmonary bioavailability. 
Our protocol included an arm with the additional 2 puffs from a pMDI administered in addition to the 5,000 $\mu \mathrm{g}$ nebulizer dose. The magnitude of an additional dose, from the $200 \mu \mathrm{g}$ dose from the pMDI ( $4 \%$ increases) was deemed not to pose an additional risk when administered to patients with COPD who were critically ill. A positive correlation was previously reported by scintigraphic studies between $\mathrm{FEV}_{1}$ and the depth of aerosol penetration into the lungs of patients with different obstructive lung diseases. ${ }^{42,43}$ Pavia et $\mathrm{al}^{44}$ demonstrated that, for subjects with COPD, the depth of aerosol deposition is directly related to $\mathrm{FEV}_{1}$. So, when $\mathrm{FEV}_{1}$ was increased through prenebulization, 2 pMDI puffs before dose administration, deeper penetration of subsequently administered medical aerosol particles was achieved as reported by Labiris and Dolovich. ${ }^{1}$ This could be the reason for our relatively higher USAL0.5 with the large spacer plus the pMDI.

Previous in vitro comparisons during conventional mechanical ventilation reported a $35 \%$ higher inhaled dose with a large spacer compared with the T-piece. ${ }^{5}$ In contrast, we found a $<5 \%$ difference between the 2 adapters during single-limb NIV. With the single-limb circuit, placement of the chamber between the fixed orifice and patient airway requires patients to exhale through the spacer, purging much of the aerosol collecting in the reservoir. Reduced aerosol delivered with the T-piece associated with the shorter distance between the aerosol-generating mesh and the opposing interior wall of the adapter, which is half the distance of the large spacer. This is consistent with our observation of condensed aerosol droplets forming within the T-piece during administration. The relatively higher USAL $0.5 \%$ of the large spacer plus pMDI in addition to the comparable USAL24\% compared with the large spacer showed that the 2 pMDI salbutamol puffs showed a trend toward increasing pulmonary bioavailability (USAL0.5\%) of the subsequent nebulized medication but not the systemic absorption (USAL24\%). This small improvement might be of no importance in a patient who has stable mild or moderate obstructive lung disease, in which most of the delivered nebulized medication, if needed, reaches the plateau of action. ${ }^{45}$ However, in patients who are critically ill, exacerbated, and on ventilation, any increase in lung delivery may be of importance. The addition of 2 pMDI puffs added only 30-60 s to the treatment time, and the cost of adding the short-acting bronchodilator pMDI to the nebulizer was minimal because all of the enrolled subjects had been prescribed a pMDI with a short-acting bronchodilator before initiation of NIV.

\section{Limitations of the Study}

During mechanical ventilation at our institution, it is not customary to interrupt ventilatory support to measure forced expiratory maneuvers, consequently, spirometry pre- and postbronchodilator administration was not measured. No significance difference was found between the T-piece and the large spacer in USAL0.5\%, USAL24, and salbutamol entrained on the filter. This was consistent with previous in vitro data that reported no significance difference between fine particle dose $(\leq 5 \mu \mathrm{m}$ and $\leq 3 \mu \mathrm{m})$ of the vibrating mesh nebulizer with both the T-piece and the large spacer. ${ }^{14}$ The coefficient of variation for urinary salbutamol was reported to be relatively high, as seen in our results. ${ }^{46}$ This and the expected $35 \%$ intra- and interpatient variability contributed to the lack of statistical significance in our small patient population. However, these data can now be used to appropriately size future studies. The small mean increase in salbutamol delivery to the lungs with the large spacer and the pMDI was hypothesized to be due to enhanced depth of aerosol penetration into the lungs. However, the use of prebronchodilation before nebulization of other drugs targeted to more peripheral airways may have a more pronounced benefit. Our findings of greater bioavailability at $0.5 \mathrm{~h}$ with administration of pMDI bronchodilator prenebulization merit further evaluation of the effects on lung function of additional pMDI doses.

\section{Conclusions}

Salbutamol delivery from a vibrating mesh nebulizer in subjects with COPD who were on single-limb NIV was equally efficient with a T-piece and a large spacer. Administration of a bronchodilator with a pMDI before nebulization showed a trend toward increasing bioavailability at $0.5 \mathrm{~h}$, which suggested that a preliminary bronchodilator dose prenebulization could improve the inhaled salbutamol fraction delivered.

\section{ACKNOWLEDGMENT}

We thank James B Fink PhD FAARC for providing help in English editing and aerosol science expertise for the development of the manuscript.

\section{REFERENCES}

1. Labiris NR, Dolovich MB. Pulmonary drug delivery. Part I: physiological factors affecting therapeutic effectiveness of aerosolized medications. Br J Clin Pharmacol 2003;56(6):588-599.

2. Lourenço RV, Loddenkemper R, Carton RW. Patterns of distribution and clearance of aerosols in patients with bronchiectasis 1, 2. Am Rev Respir Dis 1972;106(6):857-866.

3. Laube BL, Swift DL, Wagner HN Jr, Norman PS, Adams GK III. The effect of bronchial obstruction on central airway deposition of a saline aerosol in patients with asthma. Am Rev Respir Dis 1986; 133(5): 740-743.

4. Galindo-Filho VC, Ramos ME, Rattes CS, et al. Radioaerosol Pulmonary Deposition Using Mesh and Jet Nebulizers During Noninvasive Ventilation in Healthy Subjects. Respir Care 2015;60(9):12381246. 
5. Harvey CJ, O'Doherty MJ, Page CJ, Thomas SH, Nunan TO, Treacher DF. Effect of a spacer on pulmonary aerosol deposition from a jet nebuliser during mechanical ventilation. Thorax 1995;50(1):50-53.

6. Boukhettala N, Porée T, Diot P, Vecellio L. In vitro performance of spacers for aerosol delivery during adult mechanical ventilation. J Aerosol Med Pulm Drug Deliv 2015;28(2):130-136.

7. Ehrmann S, Mercier E, Vecellio L, Ternant D, Paintaud G, Dequin PF. Pharmacokinetics of high-dose nebulized amikacin in mechanically ventilated healthy subjects. Intensive Care Med 2008;34(4): 755-762.

8. Boukhettala N, Cabrera M, Diot P, Porée T, Vecellio L. In vitro evaluation of a new spacer for pMDI and nebulizers in mechanical ventilation. Eur Respir J 2012;40(Suppl 56):P2012.

9. Boukhettala N, Porée T, Diot P, Vecellio L. Study of the performances of a new spacer in adult mechanical ventilation. Eur Respir J 2013;42(Suppl 57):P3861.

10. Hassan A, Rabea H, Hussein RRS, et al. In-vitro characterization of the aerosolized dose during non-invasive automatic continuous positive airway pressure ventilation. Pulm Ther 2016;2(1):115-126.

11. ElHansy MHE, Boules ME, Farid H, et al. In vitro aerodynamic characteristics of aerosol delivered from different inhalation methods in mechanical ventilation. Pharm Dev Technol 2017;22(6):844-849.

12. Sidler-Moix AL, Di Paolo ER, Dolci U, Berger-Gryllaki M, Cotting J, Pannatier A. Physicochemical aspects and efficiency of albuterol nebulization: comparison of three aerosol types in an in vitro pediatric model. Respir Care 2015;60(1):38-46.

13. Dhand R, Tobin MJ. Inhaled bronchodilator therapy in mechanically ventilated patients. Am J Respir Crit Care Med 1997;156(1):3-10.

14. Harb HS, Elberry AA, Rabea H, Fathy M, Abdelrahim MEA. Is Combihaler usable for aerosol delivery in single limb non-invasive mechanical ventilation? J Drug Deliv Sci Technol 2017;40:28-34.

15. Fink JB, Dhand R, Grychowski J, Fahey PJ, Tobin MJ. Reconciling in vitro and in vivo measurements of aerosol delivery from a metered-dose inhaler during mechanical ventilation and defining efficiency-enhancing factors. Am J Respir Crit Care Med 1999;159(1):63-68.

16. Hassan A, Salah Eldin R, Abdelrahman MM, Abdelrahim ME. In-vitro/ in-vivo comparison of inhaled salbutamol dose delivered by jet nebulizer, vibrating mesh nebulizer and metered dose inhaler with spacer during non-invasive ventilation. Exp Lung Res 2017;43(1):19-28.

17. Newman SP, Chan HK. In vitro/in vivo comparisons in pulmonary drug delivery. J Aerosol Med Pulm Drug Deliv 2008;21(1):77-84.

18. Hussein RRS, M A Ali A, Salem HF, Abdelrahman MM, Said ASA, Abdelrahim MEA. In vitro/in vivo correlation and modeling of emitted dose and lung deposition of inhaled salbutamol from metered dose inhalers with different types of spacers in noninvasively ventilated patients. Pharm Dev Technol 2017;22(7):871-880.

19. Abdelrahim ME. Emitted dose and lung deposition of inhaled terbutaline from Turbuhaler at different conditions. Respir Med 2010; 104(5):682-689.

20. Ali A, Abdelrahim M. Modeling and Optimization of Terbutaline Emitted from a Dry Powder Inhaler and Influence on Systemic Bioavailability Using Data Mining Technology. J Pharm Innov 2014;9(1):38-47.

21. Sarhan RM, Elberry AA, Abdelwahab NS, Rabea H, Salem MN, Abdelrahim MEA. Effect of a nebulizer holding chamber on aerosol delivery. Respir Care 2018 [Epub ahead of print].

22. Saeed H, Ali AMA, Elberry AA, Eldin AS, Rabea H, Abdelrahim MEA. Modeling and optimization of nebulizers' performance in noninvasive ventilation using different fill volumes: Comparative study between vibrating mesh and jet nebulizers. Pulm Pharmacol Ther 2018;50:62-71.

23. Saeed H, Mohsen M, Salah Eldin A, et al. Effects of fill volume and humidification on aerosol delivery during single limb non-invasive ventilation. Respir Care 2018 (In Press).
24. Abdelrahim ME. Aerodynamic characteristics of nebulized terbutaline sulphate using the Andersen Cascade Impactor compared to the Next Generation Impactor. Pharm Dev Technol 2011;16(2):137-145.

25. Abdelrahim ME, Chrystyn H. Aerodynamic characteristics of nebulized terbutaline sulphate using the Next Generation Impactor (NGI) and CEN method. J Aerosol Med Pulm Drug Deliv 2009;22(1):19-28.

26. Moustafa IOF, ElHansy MHE, Al Hallag M, Fink JB, Dailey P, Rabea H, Abdelrahim MEA. Clinical outcome associated with the use of different inhalation method with and without humidification in asthmatic mechanically ventilated patients. Pulm Pharmacol Ther 2017; 45:40-46.

27. Saeed H, Elberry AA, Eldin AS, Rabea H, Abdelrahim MEA. Effect of nebulizer designs on aerosol delivery during non-invasive mechanical ventilation: a modeling study of in vitro data. Pulm Ther 2017;3(1):233-241.

28. Saeed H, Mohsen M, Fink JB, et al. Fill volume, humidification and heat effects on aerosol delivery and fugitive emissions during noninvasive ventilation. J Drug Deliv Sci Technol 2017;39:372-378.

29. ElHansy MHE, Boules ME, El Essawy AFM, et al. Inhaled salbutamol dose delivered by jet nebulizer, vibrating mesh nebulizer and metered dose inhaler with spacer during invasive mechanical ventilation. Pulm Pharmacol Ther 2017;45:159-163.

30. Abdelrahim ME, Plant P, Chrystyn H. In-vitro characterisation of the nebulised dose during non-invasive ventilation. J Pharm Pharmacol 2010;62(8):966-972.

31. Madney YM, Fathy M, Elberry AA, Rabea H, Abdelrahim MEA. Nebulizers and spacers for aerosol delivery through adult nasal cannula at low oxygen flow rate: An in-vitro study. J Drug Deliv Sci Technol 2017;39:260-265.

32. Berlinski A, Willis JR. Albuterol delivery by 4 different nebulizers placed in 4 different positions in a pediatric ventilator in vitro model. Respir Care 2013;58(7):1124-1133.

33. Ari A, Harwood RJ, Sheard MM, Fink JB. Pressurized metered-dose inhalers versus nebulizers in the treatment of mechanically ventilated subjects with artificial airways: an in vitro study. Respir Care 2015; 60(11):1570-1574.

34. Rabea H, Ali AM, Salah Eldin R, Abdelrahman MM, Said AS, Abdelrahim ME. Modelling of in-vitro and in-vivo performance of aerosol emitted from different vibrating mesh nebulisers in noninvasive ventilation circuit. Eur J Pharm Sci 2017;97:182-191.

35. Pallin M, Naughton MT. Noninvasive ventilation in acute asthma. J Crit Care 2014;29(4):586-593.

36. Moustafa IOF, Ali MRA, Al Hallag M, Rabea H, Fink JB, Dailey P, Abdelrahim MEA. Lung deposition and systemic bioavailability of different aerosol devices with and without humidification in mechanically ventilated patients. Heart Lung 2017;46(6):464-467.

37. Hindle M, Chrystyn H. Determination of the relative bioavailability of salbutamol to the lung following inhalation. Br J Clin Pharmacol 1992;34(4):311-315.

38. Mazhar SH, Ismail NE, Newton DA, Chrystyn H. Relative lung deposition of salbutamol following inhalation from a spacer and a Sidestream jet nebulizer following an acute exacerbation. Br J Clin Pharmacol 2008;65(3):334-337.

39. Evans ME, Walker SR, Brittain RT, Paterson JW. The metabolism of salbutamol in man. Xenobiotica 1973;3(2):113-120.

40. AlQuaimi M, Fink J, Ari A. Efficiency of different aerosol devices and masks during noninvasive positive pressure ventilation in a simulated adult lung model. J Respir Med Lung Dis 2017;2(3):1018.

41. Chrystyn H. Methods to identify drug deposition in the lungs following inhalation. Br J Clin Pharmacol 2001;51(4):289-299.

42. Ilowite JS, Gorvoy JD, Smaldone GC. Quantitative deposition of aerosolized gentamicin in cystic fibrosis1-3. Am Rev Respir Dis 1987;136:1445-1449. 
43. Dolovich M, Nahmias C, Coates G. Unleashing the PET: 3D imaging of the lung. Respiratory drug delivery VII niological, pharmaceutical, clinical and regulatory issues relating to optimized drug delivery by aerosol 2000;215-230.

44. Pavia D, Thomson ML, Clarke SW, Shannon HS. Effect of lung function and mode of inhalation on penetration of aerosol into the human lung. Thorax 1977;32(2):194-197.
45. Buck H, Parry-Billings M. Discriminating measures of bronchodilator drug efficacy and potency. Br J Clin Pharmacol 2001; 52(3):245-253.

46. Clark DJ, Lipworth BJ. Effect of multiple actuations, delayed inhalation and antistatic treatment on the lung bioavailability of salbutamol via a spacer device. Thorax 1996;51(10): 981-984.

This article is approved for Continuing Respiratory Care Education credit. For information and to obtain your CRCE

(free to AARC members) visit 